\title{
Galactic and extragalactic giant molecular clouds and massive star clusters: counting and analysis
}

\author{
A. S. Hojaev ${ }^{1,2}$ \\ ${ }^{1}$ National university of Uzbekistan \\ Vuzgorodok, Tashkent, Uzbekistan \\ ${ }^{2}$ Ulugh Beg Astronomical Institute, Uzbek Academy of Sciences \\ Astronomicheskaya , 33, Tashkent, 100052, Uzbekistan \\ email: hojaev@yahoo.com
}

\begin{abstract}
Comparative analysis of GMS and MSC is presented based on data collected on these objects in the Galaxy and other galaxies. The model is proposed of gravitational instability of GMC and MSC system. Some implications are discussed.
\end{abstract}

Keywords. Galaxy: structure, Galaxy: disk, (Galaxy:) open clusters and associations: general, Galaxy: kinematics and dynamics, ISM: structure, ISM: clouds, galaxies: structure, galaxies: ISM, galaxies: star clusters, surveys

\section{Introduction}

Massive star clusters (MSC), especially super star clusters and young massive star clusters, are unique objects in the Universe. The deep study of MSC together with their predecessors - giant molecular clouds (GMC) resides among the most demanded tasks in modern astronomy. The instrumentation and observing technology existed already permit to find and study GMC and MSC not only within but also outside of the Galaxy. Thus, a few dozens of GMC were scrutinized in the Milky Way (Solomon et al. 1987, Grabelsky et al. 1988 , Bronfman et al. 1989 , Heyer et al. 2009, Draine 2011, etc.) and hundreds were revealed in outer galaxies (Wilson \& Reid 1991, Taylor et al. 2000, Young 2001,Engargiola et al. 2003,Rosolowsky et al. 2007,Bolatto et al. 2008,Pineda et al. 2009,Gratier et al. 2012,Colombo et al. 2014,Kirk et al. 2015, etc.). At the same time more than half of thousand MSC including young population was found in the Galaxy and in nearby galaxies (Larsen 1999, McLaughlin \& Marel 2005, etc.) Evidently GMC should be closely associated with cold gas-dust clouds, particularly with HI ones and apparently have to play a key-role in MSC forming processes as well as they all together in the kinematics and dynamics of the host galaxy in a whole. Therefore the counting (or census) and survey of the GMC and MSC populations are still important.

\section{Results and discussion}

In an attempt to address at least some aspects of the physics and evolution of the GMC and MSC, their interplay and impact on the dynamics and evolution of galaxies we drafted a consolidated composite catalogs of GMC that could be observed from the Earth based on the recent data from DB VizieR, SIMBAD at CDS as well as original papers, reports and other publications were used. Catalogs contain the coordinates, sizes, distances, masses as well as some other physical parameters (such as density, temperature, 
radial velocity, etc.) that are available for the different clouds. They include more than 40 galactic GMC and few hundreds of GMC and super GMC in other galaxies observed in CO lines and detected up to the present. At the same time Larsen (1999) found 551 Young MSC in 21 nearby galaxies, McLaughlin \& Marel (2005) identified 153 MSC that can be resolved in the Galaxy, LMC, SMC and the Fornax dwarf spheroidal, Baumgardt et al.(2013) reported on 320 MSC in LMC. A preliminary analyses of the data has shown that in case of very young or just emerging MSC they are closely connected with GMS. Older MSC loose the connection with GMC of their origin: the formed MSC, especially with O-B supergiants that blow gas, disrupt surrounding GMC. The lifetime of GMC as well as the star formation efficiency and rate in GMC which are predictable relatively high were estimated by Murray (2011). Using these data the dynamic model of formation and evolution of GMC and MSC system based on gravitational instability develops and numerically calculated. The first results are compared with data of other investigations. The completion and improvement the catalog data are also in progress.

\section{Conclusion and implications}

The data on GMC and MSC were gathered in composite catalogs and the comparative analysis was made. The model of gravitational instability of GMC and MSC is proposed and numerically computed. As implication of the project we prepared the complex program for future observations of GMC and MSC using the 70 meter Suffa cm-submm radio telescope and other large facilities to cover the lack of observed physical parameters. This project has been supported in part by grant OT-F2-57 funded by the CCSTD at CM of RUz.

\section{References}

Solomon, P. M., Rivolo, A. R., Barrett, J., \& Yahil, A. 1987, ApJ, 319, 730

Grabelsky, D. A., Cohen, R. S., Bronfman, L., \& Thaddeus, P. 1988, ApJ, 331, 181

Bronfman, L., Nyman, L., \& Thaddeus, P. 1989, in: G. Winnewisser \& J. T. Armstrong (eds.), The Physics and Chemistry of Interstellar Molecular Clouds - $m m$ and Sub-mm Observations in Astrophysics, Lecture Notes in Physics(Berlin: Springer Verlag), 331, 139

Heyer, M., Krawczyk, C., Duval, J.,\& Jackson, J. M. 2009, ApJ, 699, 1092

Draine, B. T. 2011, Physics of the Interstellar and Intergalactic Medium (Princeton University Press), 540

Wilson, C. D. \& Reid, I. N. 1991, ApJ, 366, L11

Taylor, C., Huttemeister, S., Klein, U.\& Greve, A. 2000, ASP Conf. Ser., 215, 51

Young, L. M. 2001, AJ, 122, 1747

Engargiola, G., Plambeck, R. L., Rosolowsky, E.\& Blitz, L. 2001, ApJS, 149, 343

Rosolowsky, E. Keto, E., Matsushita, S.\& Willner, S. P. 2007, ApJ, 661, 830

Bolatto, A. D., Leroy, A. K.,Rosolowsky, E., Walter, F.\& Blitz, L. 2008, ApJ, 686, 948

Pineda, J. L. Ott, J., Klein, U., et al. 2009, ApJ, 703, 736

Gratier, P., Braine, J., Rodriguez-Fernandez, N. J., et al. 2012, AESA, 542, A108

Colombo, D.,Hughes, A., Schinnerer, E., et al. 2014, ApJ, 784, 32

Kirk, J. M., Gear, W. K., Fritz, J., et al. 2015, ApJ, 798, A58, 19

Larsen S. S. 1999, A\& $A S, 139,393$

McLaughlin, D. E.\& van der Marel, R. P. 2005, ApJS, 161, 304

Murray, N. 2011, ApJ, 729, 133

Baumgardt H., Parmentier G., Anders P.\& Grebel E. K. 2013, MNRAS, 430, 676 